Cite this: Dalton Trans., 2014, 43, 11855

Received 23rd April 2014, Accepted 15th May 2014

DOI: 10.1039/c4dt01191k www.rsc.org/dalton

\section{Facile synthesis and photoluminescence spectroscopy of 3D-triangular GaN nano prism islands $\uparrow$}

\author{
Mukesh Kumar, ${ }^{a}$ S. K. Pasha, ${ }^{a}$ T. C. Shibin Krishna, ${ }^{a}$ Avanish Pratap Singh, \\ Pawan Kumar, ${ }^{c}$ Bipin Kumar Gupta*c and Govind Gupta*a
}

\section{Introduction}

Recently, 3D triangular gallium nitride (GaN) nano prism islands (TGNPI) have been shown to play a seminal role in optically active nanoscale materials for several optoelectronic and optical sensing device applications. ${ }^{1,2}$ For example, GaN nano prism island based devices can be grown directly on silicon substrates. ${ }^{1}$ Fundamentally, GaN, a constituent of the III-V family, is a wide band gap nitride semiconductor with direct band gap of $3.39 \mathrm{eV}$ at room temperature. ${ }^{3} \mathrm{GaN}$ has a lower work function of $4.1 \mathrm{eV}$, high thermal stability, mechanical hardness, chemical stability with a lower electron affinity of 2.7-3.3 $\mathrm{eV}^{4}{ }^{4}$ Therefore, GaN may be a promising material for many optoelectronic devices such as laser diodes, light-emitting diodes, UV-based sensors and high-power electronics applications. Nowadays, 3D islands are formed using the heteroepitaxy technique; in primarily growth stages have very significant influences on film qualities and it is a subject of interest to explore their optical properties. The stringent criteria for high quality films have motivated intense research interest on

\footnotetext{
${ }^{a}$ Physics of Energy Harvesting Division, CSIR-National Physical Laboratory, Dr K S Krishnan Road, New Delhi-110012, India.E-mail: govind@nplindia.org, bipinbhu@yaoo.com; Fax: +91-11-4560-9310; Tel: +91-11-45608403

${ }^{b}$ Polymeric \& Soft Materials Section, CSIR-National Physical Laboratory, Dr K S Krishnan Road, New Delhi-110012, India

${ }^{c}$ Luminescent Materials and Devices Group, Materials Physics and Engineering Division, CSIR-National Physical Laboratory, Dr K S Krishnan Road,

New Delhi-110012, India

$\dagger$ Electronic supplementary information (ESI) available. See DOI: 10.1039/ c4dt01191k
}

island shaping and shape variations for developing high performance future miniature devices. ${ }^{1,5}$ However, island shaping is critically dependent on orientation of substrates and growth conditions. Currently, the topic of 3D shaped island morphologies of a nano-crystalline nature have become an important aspect of research due to their technological significance as well as their potential applications in the fabrication of 3D ordered arrays of nanoscale smart optoelectronic devices. ${ }^{5}$ For example, the photoluminescence of GaN nanostructures in the ultraviolet (UV) has been observed to be dependent on the growth direction of GaN. ${ }^{6}$ For successful design of the device, a better growth quality with a prominent nanostructure is highly desired. A variety of techniques for GaN growth have been employed including the extensively used techniques such as vapour-liquid-solid (VLS), ${ }^{7,8}$ metal-organic chemical vapour deposition (MOCVD) ${ }^{9}$ and molecular beam epitaxy (MBE). ${ }^{7,10}$

In the present investigation, the sputtering gun technique has been employed for GaN synthesis which is a relatively inexpensive and simpler technique that offers the possibility of growth at a lower substrate temperature. ${ }^{11,12}$ This technique has a different growth mechanism than evaporation deposition where substances are deposited on or forced into a lattice without chemical reaction. Generally, the temperature of the metal target is key for metal nitride formation. For the growth of shape oriented nanostructures, the catalyst is used for the growth of various types of metal nitride nanostructures such as nanocolumns or nanorods. The existence of a catalyst in the metal nitride nanostructure serves as an impurity that could be undesirable for device fabrication. The catalyst free growth of nanostructures using a sputtering gun technique 
may be possible by pre-annealing of metal/substrate systems such as gallium on a suitable substrate and is discussed in the present investigation.

In order to explore spectroscopic properties, photoluminescence (PL) is a direct optical tool to probe intrinsic material properties (electronic energy band structure, shape, strain, surface defect etc.) and the sensitivity of the nanostructure to environmental changes. The probability of a material to exhibit an intrinsic band structure and other internal/external defects have been studied previously. ${ }^{13}$ We have performed steady state and time-resolved photoluminescence (TRPL) measurements to determine the high quality, strain free material. The photoluminescence emission of GaN is mainly due to either near band gap excitonic emission or due to structural defects as well as the presence of internal/external impurities. In some previous reports, the observed PL spectra are attributed due to the presence of defect induced impurities in the sample that are involved in the synthesis process like other syntheses of $\mathrm{BN}^{14}$ Normally, impurity associated defects and donor acceptor states may be formed within the GaN band gap, leading to the PL in the visible region (yellow emission). Therefore, it is of fundamental interest to explore and analyse the structure to ensure the quality of GaN film for thin film based devices. TRPL is a non-destructive and commanding technique generally used for optical characterisation of semiconductors to explore their transition behaviour. The PL decay life-time is an important parameter related to the quality of the material and its performance which can be studied using TRPL spectroscopy and is relevant particularly to applications such as optical displays, optical sensors and clinical diagnostic and bio-imaging etc. ${ }^{14}$

Herein, we have successfully synthesized 3D TGNPI from the $\mathrm{Ga} / \mathrm{Si}(553)$ system at a low growth temperature by $\mathrm{N}_{2}{ }^{+}$ions implantation using a sputtering gun technique. Fabrication of 3D triangular GaN nano prism islands with detailed spectroscopy has not been reported in the literature previously to the best of our knowledge and forms the main novelty of our findings. TGNPI has been characterized by several techniques such as XRD (X-ray diffraction), SEM (scanning electron microscopy), Raman, FTIR (Fourier transform infrared spectroscopy), AFM (atomic force microscopy) and photoluminescence spectroscopy. In addition to this, we have also demonstrated time-resolved spectroscopy of luminescent TGNPI in nanoseconds regimes which is highly desirable for GaN based high-performance devices and ultra-fast optical sensors.

\section{Experimental}

\subsection{Growth of TGNPI}

Prior to the formation of GaN, the kinetically controlled growth of a few monolayers of Ga metal on a high index stepped $\mathrm{Si}(553)$ surface at room temperature along with residual thermal desorption has been reported earlier by our group. ${ }^{15}$ In the present investigation, the growth of $\mathrm{GaN}$ is performed by pre-depositing $\mathrm{Ga}$ on a $\mathrm{Si}(553)$ surface followed by nitridation, where initial stable (111) $6 \times 6+$ modified (331) superstructural phases were observed. This was assumed to act as a nucleation site for the growth of GaN nanostructures via nitridation. During the nitridation process, it is difficult to capture an intermediate superstructural phenomenon which is proposed in the present investigation.

\subsection{Characterization}

The formation of TGNPI on a stepped Si(553) surface has been carried out in an UHV system (Varian-VT112) equipped with Auger electron spectroscopy (AES), and low energy electron diffraction (LEED), sputtering gun and residual gas analyzer (RGA) operated at a base pressure of $5 \times 10^{-11}$ Torr. A modified Shiraki process was adopted for ex situ cleaning of the sample (p-type, B-doped, resistivity $0.5 \Omega \mathrm{cm}^{-1}$, size $\sim 20 \times 8 \times$ $\left.0.35 \mathrm{~mm}^{3}\right)$ followed by in situ annealing at $600^{\circ} \mathrm{C}(\sim 6 \mathrm{~h})$, flashing at $1100{ }^{\circ} \mathrm{C}$ (to remove the native oxide layers) and slowly cooling to room temperature (RT). The sample temperature was monitored using a W-Re (5\%-25\%) thermocouple fixed on the clamp. A homemade tantalum Knudsen cell was used to evaporate Ga metal (CERAC, 99.999\%) by circulating the current to control the evaporated material flux. Subsequent nitridation of the deposited Ga layers was carried out using a sputter ion gun with $2 \mathrm{keV}$ low energy nitrogen $\left(\mathrm{N}_{2}{ }^{+}\right)$ion (LENI) at different substrate temperatures (RT-650 ${ }^{\circ} \mathrm{C}$ ) leading to the formation of the GaN compound. However, for temperatures $>600{ }^{\circ} \mathrm{C}$ the desorption rate of Ga was quite high so the amount of GaN formation was significantly reduced. The gross structure analysis as well as the crystalline nature of all the GaN nanostructures (grown at RT, 450, $600{ }^{\circ} \mathrm{C}$ ) were carried out by a PANalytical high-resolution X-ray diffraction (HR-XRD) instrument using $\mathrm{CuK \alpha} \alpha_{1}$ radiation $(\lambda=1.5406 \AA)$ in a scattering range $(2 \theta)$ of $30-50^{\circ}$ with a scan rate of $0.02^{\circ} \mathrm{s}^{-1}$ and a slit width of $0.1 \mathrm{~mm}$. Fourier transform infrared (FTIR) spectra have been recorded on a Nicolet 5700 instrument in transmission mode and a wavenumber range $400-1000 \mathrm{~cm}^{-1}$ with a resolution of $4 \mathrm{~cm}^{-1}$ performing 32 scans. Raman studies were carried out using a Renishaw inVia Raman spectrometer with an excitation source of $514.5 \mathrm{~nm}$ wavelength. The XPS measurements of GaN/Si(553) were performed on an Omicronmultiprobe surface analysis system using a $\mathrm{MgK}_{\alpha} \mathrm{X}$-ray source (1253.6 eV). Core level (CL) spectra of Ga (3d), N (1s) have been fitted by using Shirley background and Gaussian line shape. The position of the valence band maximum was calculated by extrapolating a linear fit to the leading edge of the fitted valence band spectra. Binding energies were calibrated against the binding energy of the standard C (1s) peak at $284.6 \mathrm{eV}$ as well as gold which was deposited on a small portion of the sample and used as a standard to account for any kind of shift due to charging and other factors. Furthermore, the morphology of the as-grown GaN nanostructures (that is, confinement of carriers in low dimensional structures) with associated defects can affect the material properties. Therefore, the GaN nanostructures morphology and the associated defects can be effectively probed using AFM and PL measure- 
ments. The surface morphology of the GaN/Si(553) surface was analysed by a NT-MDT Solver Pro-Atomic Force Microscope (AFM). PL characterization was carried out using luminescence spectrometry (Edinburgh, FLSP-920) with a xenon flash lamp as the source of excitation as well as TRPL being recorded using a time correlated single photon counting technique with an Edinburgh FLSP-920 instrument equipped with a steady state and time-resolved luminescence spectrometer, using a picoseconds $\mathrm{He}-\mathrm{Cd}$ laser $(325 \mathrm{~nm})$ as the source of excitation.

\section{Results and discussion}

TGNPI were grown on a stepped $\mathrm{Si}(553)$ surface by depositing Ga metal followed by energetic $\mathrm{N}_{2}{ }^{+}$ion interaction as shown in Fig. 1(a). The actual set up used for the synthesis of GaN TGNPI is shown in ESI (see Fig. S1 $\dagger$ ). In the 2D (two dimensional) TGNPI shaping, the formation of triangular islands is proposed to form on either fcc (111) or hcp (001) which is further confirmed by gross structural analysis. The self organization of 3D-islands occurred during the in situ sputtering/ annealing process of GaN prepared on a $\mathrm{Si}(553)$ substrate. The distinct triangular islands are supposed to be uniform and aligned with sharp triangular corners which is further evidenced by an actual AFM micrograph. The origin of the triangular shape TGNPI can be explained via simple techniques: during the sputtering process, initially GaN is formed due to energetic nitridation by nitrogen ions which first diffuses through the deposited Ga surface and segregated on the surface of gallium deposited layer which converts into nuclei centres of GaN prismatic basal plane nuclei. As we know, the process is continuous where sputtering and annealing are simultaneously present under a nitrogen ion environment. Afterwards the unstable molecule of GaN further decomposes and reconstructs to form a prismatic basal plane at different nuclei through gas phase transport to form newly shaped islands. The adatoms attached to the island edges may also effectively migrate along the island peripheries especially, when the island size is small $(\sim 100 \mathrm{~nm})$ and the $\mathrm{Ga}$ adatoms diffusion is enhanced. Therefore, the island shaping is determined by the competition between the growth rate of the island edge facet
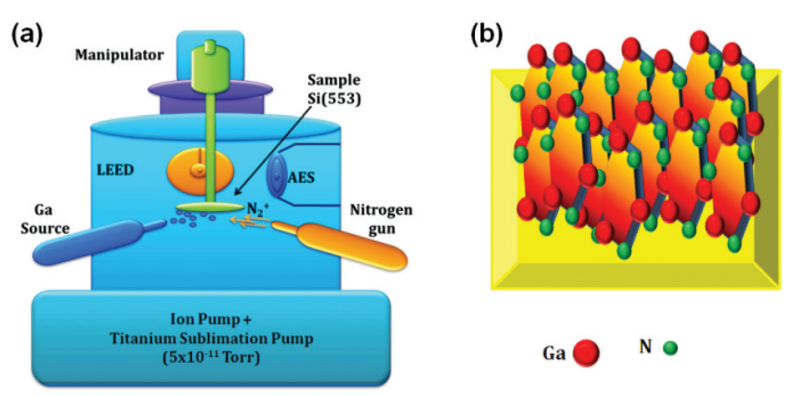

Fig. 1 (a) Schematic representation of growth system equipped with Auger electron spectroscopy, low energy electron diffraction, Ga source and a low energy nitrogen gun. (b) Cross sectional view of wurtzite GaN showing the hexagonal structure of GaN. via decomposition and re-construction of the Ga adatom. The cross section view of the wurtzite GaN phase which is the basic building block of TGNPI is shown in Fig. 1(b).

HRXRD analysis is performed to examine the crystal structure and phase purity of GaN nanostructures grown under different conditions (RT, 450 and $600{ }^{\circ} \mathrm{C}$ ). Fig. 2(a) exhibits hexagonal wurtzite GaN reflection (100), (002), (101) and (102) peaks at RT. For 450 and $600{ }^{\circ} \mathrm{C}$, the formation of TGNPI achieved where (100) and (101) planes act as basal planes for the TGNPI hexagonal wurtzite phase of GaN. The (002) plane is dominant to other planes in this case due to TGNPI growing along the (002) axis. The estimated crystal structure lattice parameters for TGNPI grown at $600{ }^{\circ} \mathrm{C}$ are $a=(3.1600 \pm$ $0.0023) \AA$ and $c=(5.2000 \pm 0.0019) \AA$. These values are in good agreement with those listed in the standard JCPDS card for wurtzite GaN (card no. 50-0792). No cubic and other impurity phase of GaN was observed. Fig. 2(b) exhibits the Raman spectra of the GaN nanostructure (RT, 450 and $600{ }^{\circ} \mathrm{C}$ ) over a wavenumber range of $500-800 \mathrm{~cm}^{-1}$ on $\mathrm{Si}(553)$ substrate at room temperature. The main GaN phonon modes are $\mathrm{A}_{1}+\mathrm{E}_{1}+$ $2 \mathrm{~B}_{1}+2 \mathrm{E}_{2}$, where $\mathrm{A}_{1}$ (LO), $\mathrm{E}_{1}$ (TO), $\mathrm{E}_{2}$ (High) and Low modes are Raman active and the $2 \mathrm{~B}_{1}$ (silent modes) are inactive. ${ }^{16}$ The observed peaks at 572, 580 and $753 \mathrm{~cm}^{-1}$ may be $\mathrm{E} 1$ (TO), $\mathrm{E}_{2}$ (High) and $\mathrm{A}_{1}$ (LO) modes, respectively, as shown in Fig. 2(b). For TGNPI grown at $600{ }^{\circ} \mathrm{C}$, a shift of $2 \mathrm{~cm}^{-1}$ towards lower wavenumber is observed which indicates the TGNPI structure contains a tensile stress of 0.4 GPa. Fig. 2(c) shows the FTIR spectra with a single peak of GaN vibration which appears at $556 \mathrm{~cm}^{-1}$ due to Ga-N stretching vibration ${ }^{17}$ in the hexagonal type GaN crystal for samples grown at RT, 450 and $600{ }^{\circ} \mathrm{C}$. Fig. 2(d) exhibits the SEM micrograph of TGNPI $\left(600^{\circ} \mathrm{C}\right)$ where the individual 3D-triangular prism morphology can be easily seen having a basal plane of dimensions around $80 \mathrm{~nm}$.

The structural morphology of the GaN nanostructures using $2 \mathrm{keV}$-LENI has been investigated by the AFM technique. The AFM scans illustrate the surface texture of the GaN/Si(553)
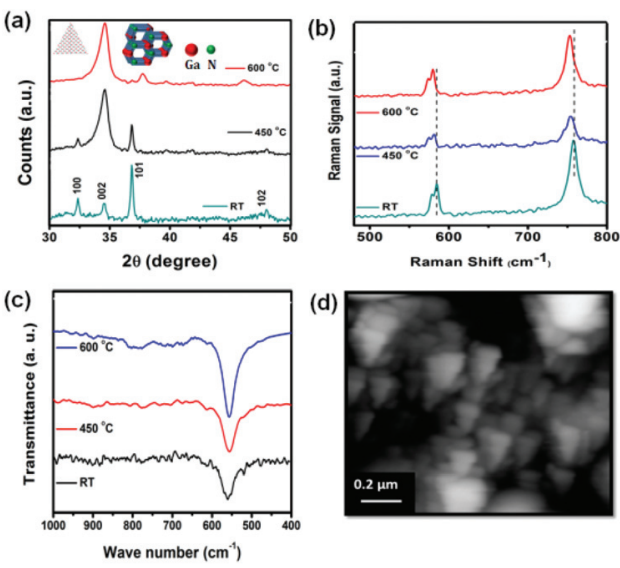

Fig. 2 (a) XRD pattern of GaN grown at RT, 450 and $600^{\circ} \mathrm{C}$ while left inset shows the growth model for TGNPI and right inset shows the cross-sectional view of wurtzite phase, (b) exhibits the Raman spectrum at different growth temperatures, (c) FTIR spectra of GaN at different growth temperatures (d) SEM micrograph of TGNPI. 
system where the surface consists of a large number of distributed symmetrical GaN nanostructures grown at RT, 450 and $600{ }^{\circ} \mathrm{C}$ (Fig. 3). For RT, the structure without the triangular morphology has been observed as shown in Fig. 3(a). For $450{ }^{\circ} \mathrm{C}$ partially nucleated triangular nanostructures were achieved as shown in Fig. 3(b). Further, when we employed $600{ }^{\circ} \mathrm{C}$ growth temperatures, uniformly developed controlled triangular nano prism islands were achieved as shown in detail in Fig. 3(c). The sequential low resolution AFM images are also shown in Fig. S2 and S3† which clearly demonstrate the TGNPI morphology. Fig. 3(d) represents the 3D image of the GaN island exhibiting the distinct and smooth sidewall faceting of TGNPI.

These GaN TGNPI consist of faceted surface enclosed by three sidewalls which results in a triangular cross-section. Although it is difficult to determine the detailed configuration of the triangular nanostructures due to the well-known convolution effect of AFM tips, and the protrusion of the nanostructures were several tens of nanometers. The average size of these GaN nanostructures can be estimated by calculating the average triangle size $<l>$, which can be defined as

$$
<l>=1 / n^{1 / 2}
$$

where $n$ is the total number of triangles per unit area. ${ }^{17}$ The average size of the triangular nanostructures $\langle l\rangle$ is found to be $\sim 75 \mathrm{~nm}$. Thus, these structures can be referred to as triangular GaN nano-prism islands (TGNPI). The formation of uniform symmetrical TGNPI were assumed to result from annealing of the $\mathrm{Ga} / \mathrm{Si}(553)$ system. On annealing at $600{ }^{\circ} \mathrm{C}$, the $\mathrm{Ga}$ develop into thermally stable islands with an induced super structural phase $\{(111) 6 \times 6+\text { modified }(331)\}^{2}$ on the $\operatorname{Si}(553)$ substrate $^{15}$ and served as nucleation sites for TGNP growth. The measured internal angles of the TGNPI were found to be around $40^{\circ}$, $70^{\circ}$, and $70^{\circ}$ where slight variations in measurements may be there due to the extraction of on-screen data. It indicates that the cross-section of a TGNPI would be an isosceles rather than an equilateral triangle, that is the observation of these
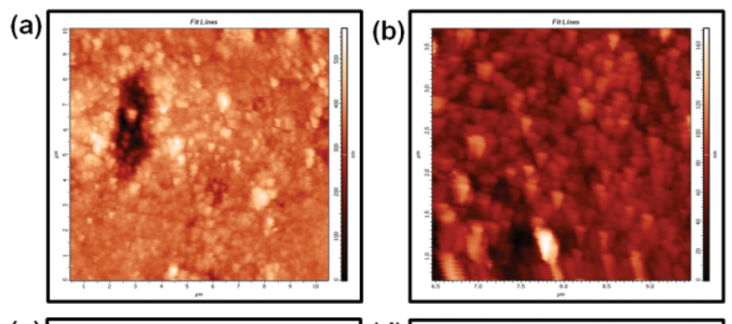

(c)
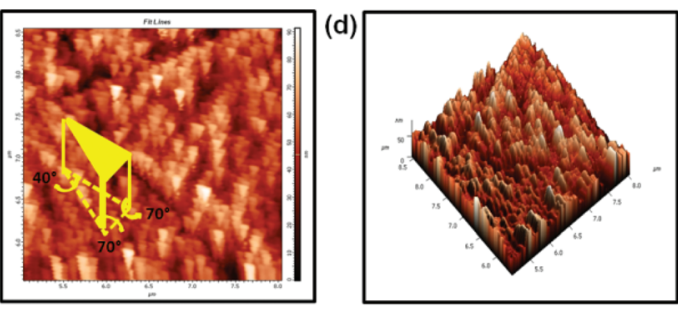

Fig. 3 AFM micrograph of GaN nanostructures grown at (a) RT (b) $450^{\circ} \mathrm{C}$ (c) $600{ }^{\circ} \mathrm{C}$ and (d) $3 \mathrm{D}$ view of TGNPI structure grown at $600{ }^{\circ} \mathrm{C}$. triangular cross-sections is not a result of viewing along the sixfold crystallographic symmetry axis which is generally observed for hexagonal wurtzite structures. Instead, the isosceles triangular cross-section is a manifestation of the two-fold symmetry. The unique nanoscale isosceles triangle of GaN might also lead to interesting carrier-confinement effects at the triangle vertices.

The deconvoluted XPS core level (CL) spectra of $\mathrm{Ga}\left(3 \mathrm{~d}_{5 / 2}\right)$ of GaN using $2 \mathrm{keV}$-LENI at different substrate temperatures is shown in Fig. 4(a-c). Deconvoluted $\mathrm{Ga}\left(3 \mathrm{~d}_{5 / 2}\right) \mathrm{CL}$ spectra (Fig. 4(a-c)) comprised three peaks, main peak at binding energy (BE) $20.2 \mathrm{eV}$ and two other smaller peaks at BE $18.4 \mathrm{eV}$ and $20.9 \mathrm{eV}$. The component of $\mathrm{BE}$ at $18.4 \mathrm{eV}$ signifies the presence of metallic Gallium adlayer $(\mathrm{Ga}-\mathrm{Ga})$ in the sample. ${ }^{1,18}$ Further, a chemical shift (1.4-1.5 eV) in the BE position of the dominating peak to the $\mathrm{BE}$ of the metallic Gallium peak has been observed in deconvoluted $\mathrm{Ga}\left(3 \mathrm{~d}_{5 / 2}\right) \mathrm{CL}$ spectra. The shift in the BE position is caused by a subtle change of the inner electron binding energy due to different chemical environments which arise from the difference between atomic valences. Thus the presence of a peak at $20.2 \mathrm{eV}$ confirms the bonding between $\mathrm{Ga}$ and $\mathrm{N}$ atoms and describes the formation of the GaN bond. ${ }^{1,18}$ The occurrence of metallic Ga in Ga $\left(3 \mathrm{~d}_{5 / 2}\right)$ CL spectra indicates the presence of Ga atoms which did not interact with the $\mathrm{N}$ species. Furthermore, there is a strong tendency for GaN surfaces to be stabilized by Ga atoms in the surface layers due to the small lattice constant and high anion-anion bond strength for GaN compared to those of conventional III-V semiconductors. ${ }^{19} \mathrm{~A}$ small peak at $\mathrm{BE}$ of $20.9 \mathrm{eV}$ could be attributed to $\mathrm{Ga}-\mathrm{O}$ bond formation in $\mathrm{GaN}$ since as-grown GaN samples were exposed to air during ex situ transfer to the XPS analysis and the presence of metallic Ga at the GaN surface also making the surface more susceptible for oxidation. Fig. 4(a) demonstrates the deconvoluted $\mathrm{Ga}\left(3 \mathrm{~d}_{5 / 2}\right)$ CL spectra for GaN using $2 \mathrm{keV}-\mathrm{LENI}$ at RT. The ratio of area under the peaks of $\mathrm{GaN} / \mathrm{Ga}\left(A_{\mathrm{GaN} / \mathrm{Ga}}\right)$ was found to be 1.7 which reveals the low extent of interaction of $\mathrm{Ga}$ atoms with
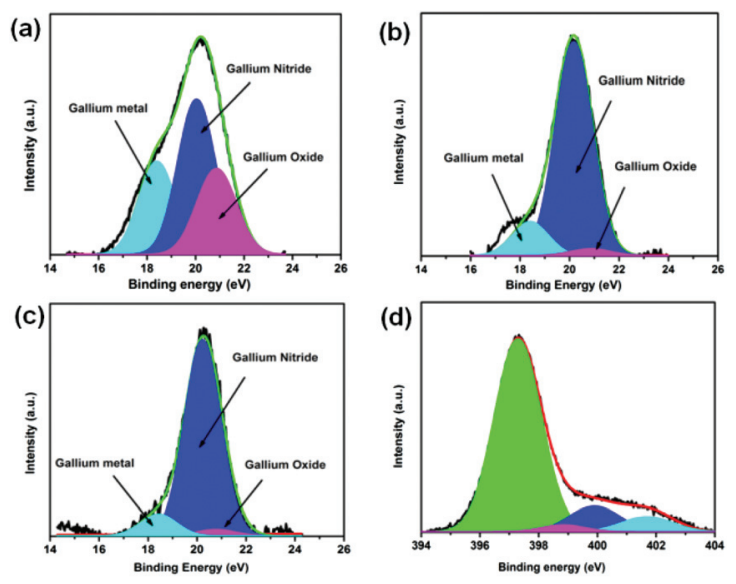

Fig. 4 Deconvoluted XPS core level spectra of Ga (3d) of GaN/Si(553) surface using $2 \mathrm{keV}$ nitrogen ions at various temperatures (a) RT, (b) $450{ }^{\circ} \mathrm{C}$, (c) $600{ }^{\circ} \mathrm{C}$ and (d) $\mathrm{N}$ (1s) spectra for GaN/Si(553) formed at $600^{\circ} \mathrm{C}$ using $2 \mathrm{keV}$ nitrogen ions. 
$\mathrm{N}$-ions at RT. Fig. 4(b) shows the formation of GaN using $2 \mathrm{keV}$-LENI at $450{ }^{\circ} \mathrm{C}$ where a significant reduction in the contribution of $\mathrm{Ga}-\mathrm{Ga}$ and $\mathrm{Ga}-\mathrm{O}$ was observed. Further the value of $A_{\mathrm{GaN} / \mathrm{Ga}}$ was found to be 6.4, which signifies a higher nitridation at $450{ }^{\circ} \mathrm{C}$ than RT. Since atomic diffusion is characteristic of the thermal process. Therefore, annealing of the substrate at $450{ }^{\circ} \mathrm{C}$ provides thermal energy to the $\mathrm{Ga}$ atoms and the implanted light atoms that are mobile at elevated temperature can diffuse into the mother lattice. Also, there is less probability of thermal decomposition of $\mathrm{GaN}$ at low temperature. These considerations may be the reason for a higher nitridation at $450{ }^{\circ} \mathrm{C}$ than RT. On further increasing the nitridation temperature to $600{ }^{\circ} \mathrm{C}$, the value of $A_{\mathrm{GaN} / \mathrm{Ga}}$ increased to 9.8 which indicates significant interaction of $\mathrm{Ga}$ atoms with $\mathrm{N}$-ions. On further increasing the nitridation temperature $\left(>600{ }^{\circ} \mathrm{C}\right)$ a significant reduction in the Ga coverage was observed due to the desorption of Ga from the $\mathrm{Si}(553)$ surface. XPS CL spectra analysis revealed that $2 \mathrm{keV}-\mathrm{LENI}$ at $600{ }^{\circ} \mathrm{C}$ provides the required energy for the high quality growth of GaN. Further, the contribution of Ga in Ga-rich GaN can be controlled by optimizing the substrate temperature. The deconvoluted CL-N (1s) spectra has also been shown for $2 \mathrm{keV}-\mathrm{LENI}$ at $600{ }^{\circ} \mathrm{C}$ which contains a peak at $397.0 \mathrm{eV}(\mathrm{GaN})$ along with three small peaks at $399 \mathrm{eV}(\mathrm{N}-\mathrm{O}-\mathrm{Ga}), 400 \mathrm{eV}(\mathrm{N}-\mathrm{H})$ and $401.7 \mathrm{eV}(\mathrm{N}-\mathrm{C})$. The peaks corresponding to $\mathrm{N}-\mathrm{H} \& \mathrm{~N}-\mathrm{C}$ components might have occurred due to surface $\mathrm{H}$ - and C-adsorption, during ex situ sample transfer process.

The energy structure of $\mathrm{GaN} / \mathrm{Si}(553)$ was identified by valence band (VB) spectra. The corresponding VB spectra are shown in Fig. S4(a-c) (ESI $\dagger)$. There were two dominant structures except for the core level $\mathrm{Ga}(3 \mathrm{~d})$ peak, in the valence band spectra. The Gaussian fitted peaks of these two structures were centered at $\mathrm{BE}$ of $5.7 \mathrm{eV}$ and $10 \mathrm{eV}$. The $5.7 \mathrm{eV}$ feature is mainly due to nitrogen p-derived, and the peak at $10 \mathrm{eV}$ is associated with hybridized $\mathrm{Ga}(\mathrm{s})$ and $\mathrm{N}(\mathrm{p})$ states and the location of these two maxima agree well with the reported values and major density of state (DOS) features predicted in the band structure calculations..$^{20,21}$ The RT grown GaN Fig. S4(a) $\dagger$ as well as $450{ }^{\circ} \mathrm{C}$ grown samples Fig. $\mathrm{S} 4(\mathrm{~b}) \dagger$ have a slightly reduced intensity of nitrogen p-derived structure than the feature associated with hybridized Ga (s) and $N(p)$ states. While, these features have comparable intensity with the $600{ }^{\circ} \mathrm{C}$ grown sample Fig. S4(c). $\dagger$ As the Ga-component was different in various $\mathrm{GaN}$ samples and reduces with an increase in growth temperature up to $600{ }^{\circ} \mathrm{C}$ as shown in Fig. $S 4(a-c) . \dagger$ It suggests that the low temperature grown GaN sample exhibits a more nitrogen depleted surface which changes the line-shape of the valence band. Therefore, the feature associated with hybridized $\mathrm{Ga}(\mathrm{s})$ and $\mathrm{N}(\mathrm{p})$ states remains intact, while the nitrogen $\mathrm{p}$-derived structure reduced in intensity with higher nitrogen depleted surface in GaN which is further evidenced by photoluminescence spectroscopy where the PL intensity of TGNPI is higher compared to RT \& 450 grown samples. The tail of the valence band spectra shows the existence of surface states below the unidentified accurate position of the VB maximum (VBM) where some energy states lie below the zero binding energy. If a significant number of occupied states in the band gap of GaN exist at the surface, photoemission could occur from these states and would cause the valence band leading edge to appear falsely and make it difficult to determine the position of VBM. These observed states, existing below the VBM, can result from various factors such as a small gallium component in the GaN sample, differences in film/surface preparation, defects, strain and contaminants. It is interesting to note that the reported $\mathrm{BE}$ values of $\mathrm{CL} \mathrm{Ga}(3 \mathrm{~d})$-VBM for GaN vary in the range of 17.1-18.4 eV and the VBM of clean GaN surfaces located in the 2.4-2.7 eV range below the Fermi level. ${ }^{22}$ However, CL-VBM values are independent of the measurement artifact effects due to the existence of surface states. ${ }^{22,23}$ The leading edge of the Gaussian fit peak comes around $2.5 \mathrm{eV}$ Fig. S4(c) $\dagger$ suggesting the position of VBM and the CL-VBM values to be $17.7 \mathrm{eV}$.

Being able to visualize the optoelectronic devices on the basis of optical properties of the TGNPI, the photoluminescence studies were carried out. Fig. 5(a-c) shows the photoluminescence emission spectrum of GaN nanostructures upon $325 \mathrm{~nm}$ excitation wavelength at room temperature. Fig. 5(a) displays the photoluminescence emission spectra of GaN grown at RT, 450 and $600{ }^{\circ} \mathrm{C}$. The PL results reveal that the intensity of the PL spectrum of TGNPI grown at $600{ }^{\circ} \mathrm{C}$ is $\sim 3$ and 7 times higher than 450 and RT grown samples respectively. The observed higher PL intensity in the case of TGNPI grown at $600{ }^{\circ} \mathrm{C}$ originates due to the higher crystallinity achieved which creates more electrons and holes for recombination as compared to RT and $450{ }^{\circ} \mathrm{C}$ grown samples. The availability of the higher electron hole pair density in GaN nanostructures depends upon the contributions of GaN as well as N-p derivatives which control the recombination process. In the present case, the sample grown at $600{ }^{\circ} \mathrm{C}$ has less $\mathrm{N}-\mathrm{p}$ derivative as compared to RT \& $450{ }^{\circ} \mathrm{C}$. The presence of higher p-derivative quenches the luminescence which can be easily
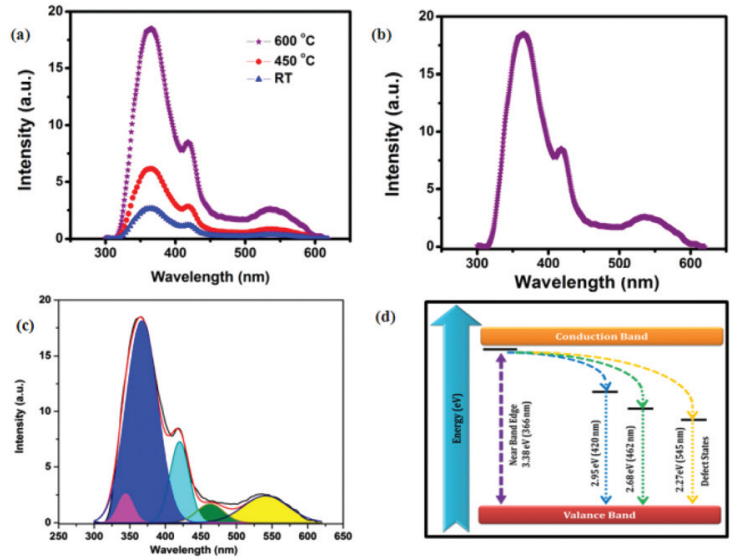

Fig. 5 (a) Shows the photoluminescence emission spectra of GaN grown at different temperatures. (b) PL emission spectrum of TGNPI grown at $600{ }^{\circ} \mathrm{C}$. (c) Deconvoluted Gaussian residuals peaks of TGNPI and (d) proposed energy level diagram based on Gaussian fitting. 
seen in Fig. 5(a). The PL results are in good correlation with the observed VB spectra as shown in Fig. S4. $\dagger$

In order to explore the detailed mechanism of the photoluminescence phenomena of TGNPI grown at $600{ }^{\circ} \mathrm{C}$, we plot the separate PL spectrum of TGNPI grown at $600{ }^{\circ} \mathrm{C}$ and deconvoluted the spectrum which are shown in Fig. 5(b) and 5(c). Mainly three dominant Gaussian fit peak structures centred at $366 \mathrm{~nm}, 420 \mathrm{~nm}$ and $545 \mathrm{~nm}$ have been observed besides the Gaussian residuals peaks around $345 \mathrm{~nm}$ and $461 \mathrm{~nm}$ as shown in Fig. 5(c). The pronounced peak feature centred at $366 \mathrm{~nm}$ corresponds to the near-bandgap emission (NBE) for the GaN where the peak position is at a characteristic wavelength corresponding to the band-edge emission. ${ }^{24}$ The contributions to the NBE that result in the broadening of the emission peak, can result from the excitons bound to neutral shallow donor impurities, point defects and structural defects such as stacking faults from the bottom interface of the nanostructures. $^{25}$ The residual peak feature results from the asymmetry in the PL peak structure that has emission around $345 \mathrm{~nm}$, is blue-shifted relative to the GaN band-edge PL at $366 \mathrm{~nm}$. The possibility of a blue shift peak feature may be due to the emission from the triangle vertices of TGNPI where the carriers are confined leading to an increased band gap on the basis of quantum confined states. Further, there was a small contribution of $\mathrm{Ga}$ at the surface of GaN nanostructures as observed in the XPS spectra as shown in Fig. 4(d), which makes the surface more susceptible to oxidation. Perhaps oxidation of the surface leading to the formation of surface trap states, might also contribute to the emission in the spectral range of the blue-shifted PL. The sub-band-gap emission (SBE) is shown by the dominant sharp peak feature around $420 \mathrm{~nm}$. In the case of silicon substrate, the possible cause for SBE emission may be attributed to the donor-acceptor pair recombination (DAP) ${ }^{25}$ due to inter-diffusion of silicon atoms into the GaN nanostructures that has an n-type doping behaviour where the acceptor state is most likely related to unidentified intrinsic defects in the GaN lattice. In addition, there is a spectral feature consisting of the dominant broad peak structure observed at higher wavelength centred at $545 \mathrm{~nm}$ and the residual peak feature around $461 \mathrm{~nm}$. The broad feature around $545 \mathrm{~nm}$, which is yellow luminescence, is a well-known problem in GaN films. ${ }^{26}$ These features result from the structural imperfections that are almost six orders of magnitude weaker with respect to the NBE feature which is characterized by the qualitative superiority of TGNPI with low defects. The band edge as well as defect states associated PL phenomena and their transitions can be easily understood by the proposed energy level diagram of TGNPI as shown in Fig. 5(d).

Time-resolved photoluminescence spectroscopy (TRPL) is a non destructive and very powerful tool to explore the photoluminescence transition. The PL decay life-time is an important parameter related to the quality of the material and its performance, which can be studied using TRPL spectroscopy, and is relevant particularly to applications such as optical displays, optical sensors, clinical diagnostics and bio-imaging. To study the recombination mechanism of the emission, we have carried out time-resolved PL by excitation with a laser line at $325 \mathrm{~nm}$ for all samples (grown at RT, 450 and $600{ }^{\circ} \mathrm{C}$ ). It is well established that the TRPL measurements on individually dispersed and as-synthesized GaN nanostructures give a PL decay time in the range of $\sim 200$ ps to about $\sim 2.7$ ns dependent on the temperature and the dimensions of the nanostructure. ${ }^{10}$ Fig. S5† represents the decay profile of samples grown at RT, 450 and $600{ }^{\circ} \mathrm{C}$. The life-time increases with respect to the growth temperature of the GaN nanostructures. Time-resolved decay profiles basically represent the efficiency of the radiative recombination which is directly proportional to the decay time of the particular transition either it is associated with the impurity or ion and defects. The obtained TRPL results are consistent with the acquired PL data. It is clearly evident that the PL intensity increases with temperature because the recombination part is enhanced with respect to temperature, as a result it can be easily understood that the life-time quenches for RT and $450{ }^{\circ} \mathrm{C}$ where TGNPI either didn't grow or only partially grew. Fig. 6(a) shows the PL decay profile of the TGNPI grown at $600{ }^{\circ} \mathrm{C}$. The decay was recorded for the TGNPI transition at $366 \mathrm{~nm}$ emission obtained at room temperature upon $325 \mathrm{~nm}$ excitation wavelength of HeCd laser by a time-correlated singlephoton counting technique. The life-time data of TGNPI were very well fitted to a triple-exponential function as:

$$
I(t)=A_{1} \exp \left(-t / \tau_{1}\right)+A_{2} \exp \left(-t / \tau_{2}\right)+A_{3} \exp \left(-t / \tau_{3}\right)
$$

where $\tau_{1}, \tau_{2}$ and $\tau_{3}$ are the decay life-times of the luminescence, and $A_{1}, A_{2}$ and $A_{3}$ are the weighting parameters. The observed life-times of the TGNPI are $\tau_{1} \sim 0.56 \mathrm{~ns}, \tau_{2} \sim 0.96 \mathrm{~ns}$ and $\tau_{3} \sim$ 4.86 ns as shown in Fig. 6(b).

For triple-exponential decay, the average decay time $\left(\tau_{\mathrm{av}}\right)$, was determined by the following equation:

$$
\tau_{\mathrm{av}}=\frac{A_{1} \tau_{1}^{2}+A_{2} \tau_{2}^{2}+A_{3} \tau_{3}^{2}}{A_{1} \tau_{1}+A_{2} \tau_{2}+A_{3} \tau_{3}}
$$

The average PL decay time for TGNPI was calculated as $\tau_{\text {av }} \sim 2$ ns. Such a decay time compares favorably with the PL decay time and carrier life-times observed in high quality bulk GaN threading dislocations densities below $10^{6} \mathrm{~cm}^{-2} \cdot{ }^{27,28}$ The obtained time-resolved spectroscopy results clearly demonstrate the triple exponential decay curve where the curve fitting
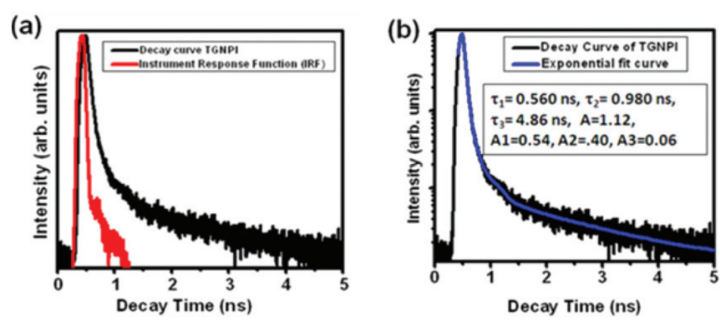

Fig. 6 (a) Shows the TRPL spectrum of the TGNPI upon $366 \mathrm{~nm}$ emission at $325 \mathrm{~nm}$ excitation wavelengths (RT), (b) exponential fitted decay curve of TGNPI while the inset shows the curve fitting generated parameters. 
generated relative contributions of different weighting parameters $A_{1}, A_{2}, A_{3}$ are $54 \%, 40 \%, 6 \%$ respectively. However, it is difficult to designate the energy states, types of defects and defect density which are associated with the obtained weighting parameters. It only reveals that three energy states are involved for such transitions corresponding to contributions of a three decay component in time resolved spectroscopy after exponential curve fitting. The cause of the three decay component present in TGNPI may be due to energy trapping in different defect states present in TGNPI structures which is explained by the proposed energy level diagram. Thus, the TRPL spectroscopy result suggests that the TGNPI is highly suitable for fast optical UV sensing/switching applications as well as high performance optoelectronic devices.

\section{Conclusions}

In conclusion, we have demonstrated the formation of TGNPI $\mathrm{GaN}$ from $\mathrm{Ga} / \mathrm{Si}(553)$ system at low growth temperature by 2 keV-LENI implantation using a sputtering gun technique. High quality TGNPI formation is achieved using $2 \mathrm{keV}$ ion energy at a substrate temperature $600{ }^{\circ} \mathrm{C}$. The morphology of the as-grown sample consists of GaN nano-prisms islands with a triangular cross-section. These TGNPI have blue shifted luminescence with a prominent GaN characteristic emission wavelength at $366 \mathrm{~nm}$ upon $325 \mathrm{~nm}$ excitation wavelength. The defects in the TGNPI are observed to be low which is shown by a six time lower intensity of defect related PL than the characteristic PL intensity corresponding to GaN. Time-resolved spectroscopy of TGNPI reveals that the nanosecond life-time holds promise for its potential application as an optoelectronic, UV sensor as well as a high performance optical switching application at the nanoscale level.

\section{Acknowledgements}

The authors gratefully acknowledge Prof. R. C. Budhani Director, CSIR-NPL, New Delhi, India for constant encouragement and support. The work is supported by CSIR network project TAPSUN (NWP-55) Inorganic Light Emitting Diode Activity.

\section{References}

1 Z. Fang and J. Kang, J. Phys. Chem. C, 2007, 111, 78897892.

2 D. V. Dinh and J. H. Yang, J. Korean Phys. Soc., 2009, 55, 202-206.

3 W. Shan, T. J. Schmidt, R. J. Hauenstein and j. J. Song, Appl. Phys. Lett., 1995, 66, 3492-3494.

4 D. V. Dinh, S. M. Kang, J. H. Yang, S. W. Kim and D. H. Yoon, J. Cryst. Growth, 2009, 311, 495-499.

5 M. D. Brubaker, P. T. Blanchard, J. B. Schlager, A. W. Sanders, A. Roshko, S. M. Duff, J. M. Gray,
V. M. Bright, N. A. Sanford and K. A. Bertness, Nano Lett., 2013, 13, 374-377.

6 A. H. Chin, T. S. Ahn, H. Li, S. Vaddiraju, C. J. Bardeen, C.-Z. Ning and M. K. Sunkara, Nano Lett., 2007, 7, 626-631.

7 T. Kuykendall, P. J. Pauzauskie, Y. Zhang, J. Goldberger, D. Sirbuly, J. Denlinger and P. Yang, Nat. Mater., 2004, 3, 524-528.

8 T. W. George, A. A. Talin, J. W. Donald, J. R. Creighton, L. Elaine, J. A. Richard and A. Ilke, Nanotechnology, 2006, 17, 5773 .

9 T. Kuykendall, P. Pauzauskie, S. Lee, Y. Zhang, J. Goldberger and P. Yang, Nano Lett., 2003, 3, 1063-1066.

10 J. B. Schlager, K. A. Bertness, P. T. Blanchard, L. H. Robins, A. Roshko and N. A. Sanford, J. Appl. Phys., 2008, 103, 124309.

11 T. Kawabata, F. Okuyamaa and M. Tanemura, J. Appl. Phys., 1991, 69, 3723-3728.

12 J. A. Taylor, G. M. Lancaster, A. Ignatiev and J. W. Rabalais, J. Chem. Phys., 1978, 68.

13 B. K. Gupta, V. Shanker, M. Arora and D. Haranath, Appl. Phys. Lett., 2009, 95, 073115.

14 A. L. M. Reddy, B. K. Gupta, T. N. Narayanan, A. A. Martí, P. M. Ajayan and G. C. Walker, J. Phys. Chem. C, 2012, 116, 12803-12809.

15 M. Kumar, S. K. Pasha and Govind, Appl. Surf. Sci., 2013, 283, 1071-1075.

16 M. Kuball, Surf. Interface Anal., 2001, 31, 987-999.

17 Govind, W. Chen, H. Wang and T. E. Madey, Phys. Rev. B: Condens. Matter, 2010, 81, 085415.

18 J. B. Metson, H. J. Trodahl, B. J. Ruck, U. D. Lanke and A. Bittar, Surf. Interface Anal., 2003, 35, 719-722.

19 T. D. Veal, P. D. C. King, P. H. Jefferson, L. F. J. Piper, C. F. McConville, H. Lu, W. J. Schaff, P. A. Anderson, S. M. Durbin, D. Muto, H. Naoi and Y. Nanishi, Phys. Rev. B: Condens. Matter, 2007, 76.

20 J. Ma, B. Garni, N. Perkins, W. L. O’Brien, T. F. Kuech and M. G. Lagally, Appl. Phys. Lett., 1996, 69, 22.

21 M.-Z. Huang and W. Y. Ching, J. Phys. Chem. Solids, 1985, 46.

22 S. W. King, C. Ronning, R. F. Davis, M. C. Benjamin and R. J. Nemanich, J. Appl. Phys., 1998, 84.

23 X. Xu, X. Liu, Y. Guo, J. Wang, H. Song, S. Yang, H. Wei, Q. Zhu and Z. Wang, J. Appl. Phys., 2010, 107, 104510.

24 K. M. A. Saron, M. R. Hashim and N. K. Allam, J. Appl. Phys., 2013, 113, 124304.

25 F. Furtmayr, M. Vielemeyer, M. Stutzmann, A. Laufer, B. K. Meyer and M. Eickhoff, J. Appl. Phys., 2008, 104.

26 K. B. Nam, M. L. Nakarmi, J. Y. Lin and H. X. Jiang, Appl. Phys. Lett., 2005, 86, 222108.

27 T. Malinauskas, R. Aleksiejūnas, K. Jarašiūnas, B. Beaumont, P. Gibart, A. Kakanakova-Georgieva, E. Janzen, D. Gogova, B. Monemar and M. Heuken, J. Cryst. Growth, 2007, 300, 223-227.

28 S. F. Chichibu, A. Uedono, T. Onuma, T. Sota, B. A. Haskell, S. P. DenBaars, J. S. Speck and S. Nakamura, Appl. Phys. Lett., 2005, 86, 021914. 